\title{
Discovery of a Positive Allosteric Modulator of the Thyrotropin Receptor: Potentiation of Thyrotropin-Mediated Preosteoblast Differentiation In Vitro $\$$
}

\author{
Susanne Neumann, Elena Eliseeva, Alisa Boutin, Elena Barnaeva, Marc Ferrer, \\ Noel Southall, David Kim, Xin Hu, Sarah J. Morgan, Juan J. Marugan, \\ and Marvin C. Gershengorn
}

\begin{abstract}
Laboratory of Endocrinology and Receptor Biology, National Institute of Diabetes and Digestive and Kidney Diseases, National Institutes of Health, Bethesda, Maryland (S.N., E.E., A.B., S.J.M., M.C.G.); and Division of Pre-Clinical Innovations, National Center for Advancing Translational Sciences, National Institutes of Health, Rockville, Maryland (E.B., M.F., N.S., D.K., X.H., J.J.M.)
\end{abstract}

Received July 18, 2017; accepted October 26, 2017

\begin{abstract}
Recently, we showed that TSH-enhanced differentiation of a human preosteoblast-like cell model involved a $\beta$-arrestin $1(\beta$-Arr 1)-mediated pathway. To study this pathway in more detail, we sought to discover a small molecule ligand that was functionally selective toward human TSH receptor (TSHR) activation of $\beta$-Arr 1. High-throughput screening using a cell line stably expressing mutated TSHRs and mutated $\beta$-Arr 1 (DiscoverX1 cells) led to the discovery of agonists that stimulated translocation of $\beta$-Arr 1 to the TSHR, but did not activate $\mathrm{G}_{\mathrm{s}}$-mediated signaling pathways, i.e., cAMP production. D3- $\beta$ Arr (NCGC00379308) was selected. In DiscoverX1 cells, D3- $\beta$ Arr stimulated $\beta$-Arr 1 translocation with a 5.1-fold greater efficacy than TSH and therefore potentiated the effect of TSH in stimulating $\beta$-Arr 1 translocation. In human U2OS-TSHR cells expressing wild-type TSHRs, which is a
\end{abstract}

model of human preosteoblast-like cells, TSH upregulated the osteoblast-specific genes osteopontin (OPN) and alkaline phosphatase (ALPL). D3- $\beta$ Arr alone had only a weak effect to upregulate these bone markers, but D3- $\beta$ Arr potentiated TSHinduced upregulation of ALPL and OPN mRNA levels 1.6-fold and 5.5-fold, respectively, at the maximum dose of ligands. Furthermore, the positive allosteric modulator effect of D3- $\beta$ Arr resulted in an increase of TSH-induced secretion of OPN protein. In summary, we have discovered the first small molecule positive allosteric modulator of TSHR. As D3- $\beta$ Arr potentiates the effect of TSH to enhance differentiation of a human preosteoblast in an in vitro model, it will allow a novel experimental approach for probing the role of TSH-induced $\beta$-Arr 1 signaling in osteoblast differentiation.

\section{Introduction}

Bone remodeling is essential for sustaining bone mass and systemic mineral homeostasis and requires a balance between bone formation by osteoblasts and bone resorption by osteoclasts (Martin et al., 2009; Siddiqui and Partridge, 2016). Parathyroid hormone (PTH) is the major regulator of bone homeostasis (Qin et al., 2004) and acts by activating the PTH 1 receptor (PTH1R), which is a G protein-coupled receptor (GPCR). The anabolic effects of PTH appear to be mediated in large part via $\mathrm{G}$ protein-independent $\beta$-arrestin 2 ( $\beta$-Arr 2 ) signaling (Gesty-Palmer and Luttrell, 2011). The TSH receptor (TSHR), like PTH1R, is a GPCR (Kleinau et al., 2011)

This research was supported by the Intramural Research Program of the National Institutes of Health National Institute of Diabetes and Digestive and Kidney Diseases [Grant Z01 DK011006] and the National Center for Advancing Translational Sciences.

https://doi.org/10.1124/jpet.117.244095.

S This article has supplemental material available at jpet.aspetjournals.org. that is expressed primarily in thyroid follicular cells. The physiologic actions of TSH have been thought to be mediated by heterotrimeric $\mathrm{G}$ protein signaling pathways, and $\mathrm{G}_{\mathrm{s}}$-mediated cAMP signaling has been considered the primary pathway in the thyroid (Latif et al., 2009). However, TSHR expression has also been reported in bone (Williams, 2011). TSHR expression in rodent osteoblasts and osteoclasts has been demonstrated, effects of TSH on bone homeostasis have been studied, and the results suggested that TSH is a fine-tuning regulator of bone homeostasis (Abe et al., 2003, 2007). Specific effects observed in rodents include: TSHR knockout causes osteoporosis and focal osteosclerosis (Abe et al., 2003), TSH stimulates osteoblastogenesis in embryonic stem cells from mice (Baliram et al., 2011), and TSH administration prevents bone loss in adult ovariectomized rats and mice (Sun et al., 2008). However, the role of TSH in human bone homeostasis is still unclear (Mazziotti et al., 2005; Giusti et al., 2007; Karga et al., 2010; Leader et al., 2014). To gain insight into the effects of TSH in human cells, we used a preosteoblast-like cell line derived from a human osteosarcoma

ABBREVIATIONS: ALPL, alkaline phosphatase; $\beta$-Arr $1, \beta$-arrestin 1 ; BSA, bovine serum albumin; ELISA, enzyme-linked immunosorbent assay; EMEM, Eagle's minimum essential medium; FBS, fetal bovine serum; GPCR, G protein-coupled receptor; HBSS, Hank's balanced salt solution; IBMX, 3-isobutyl-1-methylxanthine; OPN, osteopontin; PAM, positive allosteric modulator; PTH, parathyroid hormone; PTH1R, parathyroid hormone 1 receptor; qHTS, quantitative high-throughput screening; RT-PCR, real-time polymerase chain reaction; siRNA, small interfering RNA; TSH, thyrotropin; TSHR, TSH receptor; U2OS cells, human osteosarcoma cells. 
made to stably express wild-type TSHR, U2OS-TSHR cells (Boutin et al., 2014, 2016). U2OS cells have been shown to differentiate into osteoblasts (Salvatori et al., 2009). We demonstrated that TSH enhances differentiation of U2OSTSHR cells to osteoblasts and that this effect is partly mediated by $\beta$-arrestin 1 ( $\beta$-Arr 1) (Boutin et al., 2014).

Biased agonism, which is selective activation of one signaling pathway by a receptor that exhibits signal transduction via several pathways, has been shown for many GPCRs (Rominger et al., 2014; Wisler et al., 2014; Rankovic et al., 2016). The discovery that $\beta$-arrestins play a role in GPCR signaling has generated new strategies for drug therapies. Of note, a peptidic $\beta$-Arr 2 biased agonist for PTH1R, PTH- $\beta$ arr, has been used in elucidating the role of $\beta$-Arr signaling in PTH1R biology (Gesty-Palmer and Luttrell, 2011). The physiologic response induced by PTH- $\beta$ arr in bone is distinct from that of the conventional PTH1R agonist PTH(1-34), a peptide fragment that contains the $34 \mathrm{~N}$-terminal amino acid residues of the naturally occurring human PTH (hPTH) and that has the same biologic activity as hPTH in stimulating bone formation (Mosekilde et al., 1991). Intermittent administration of PTH(1-34) increases bone formation, but PTH(1-34) administration also causes increased bone resorption that can lead to hypercalcemia and hypercalcuria. The $\beta$-arrestin selective PTH- $\beta$ arr induces anabolic bone formation but does not increase markers of bone resorption (Gesty-Palmer et al., 2009). This example underlines that functionally selective ligands could have beneficial effects compared with the native agonists for a GPCR, and, therefore, these ligands hold promise for more effective treatments of human diseases.

Modulators of GPCR signaling exhibit little functional activity by themselves but modify the signaling by the cognate ligand. Allosteric modulators bind to a receptor at a site distinct from that of the cognate ligand (orthosteric binding site), in many cases displaying pharmacological characteristics that are different from orthosteric ligands. Therefore, allosteric modulators offer an alternate approach to gain potential therapeutic benefits (Gentry et al., 2015). Allosteric modulators may agonize (positive allosteric modulator, PAM) or antagonize (negative allosteric modulator) the activity (potency/efficacy) of orthosteric endogenous ligands (Gentry et al., 2015). PAMs, which potentiate agonist activity, have been developed for a number of receptors and ion channels (Conn et al., 2009; Burford et al., 2011; Wootten et al., 2013), including GPCRs with known ligands (O'Brien et al., 2003; Harrington and Fotsch, 2007; Langmead et al., 2008; Coopman et al., 2010; Wood, et al., 2016), and for two orphan receptors (Huang et al., 2015).

In light of the role of TSHR - $\beta$-Arr 1-mediated signaling in osteoblast differentiation (Boutin et al., 2014), we screened for small molecule $\beta$-Arr 1 signaling-selective agonists for TSHR that could be used to enhance TSH-induced osteoblast differentiation.

\section{Materials and Methods}

Culture of U2OS-TSHR Cells, DiscoverX1, and DiscoverX2 Cells. The generation of a U2OS (human osteosarcoma) cell line stably expressing wild-type TSHRs (U2OS-TSHR) using the expression vector for native human TSHR was described previously (Boutin et al., 2014). DiscoverX1 and DiscoverX2 cells, U2OS cells expressing functional tagged TSHR and functional tagged $\beta$-Arr 1 or $\beta$-Arr 2 , respectively, were purchased from DiscoverX (Fremont, CA). U2OS-TSHR cells were cultured in Eagle's minimum essential medium (EMEM) (ATCC, Manassas, VA). EMEM was supplemented with $10 \%$ fetal bovine serum (FBS) (Sigma Aldrich, St. Louis, MO), $50 \mathrm{U} / \mathrm{ml}$ penicillin, and $50 \mu \mathrm{g} / \mathrm{ml}$ streptomycin (Life Technologies Inc., Carlsbad, CA). Hygromycin B $(250 \mu \mathrm{g} / \mathrm{ml})$ (ThermoFisher Scientific, Waltham, MA) was used as a selection marker. All experiments for the measurement of osteoblast markers were performed in the medium described above, but with $0.1 \%$ bovine serum albumin (BSA) instead of 10\% FBS. For DiscoverX1 and DiscoverX2 cells, EMEM was also supplemented with $2 \mathrm{mM}$ glutamine and $500 \mu \mathrm{g} / \mathrm{ml}$ geneticin (Mediatech, Manassas, VA). Cells were cultured at $37^{\circ} \mathrm{C}$ in a humidified $5 \% \mathrm{CO}_{2}$ incubator.

Quantitative High-Throughput Screening. Quantitative highthroughput screening (qHTS) was performed against 368,816 compounds from the Molecular Libraries Small Molecule Repository at the National Center for Advancing Translational Sciences using the DiscoverX1 cells and the PathHunter $\beta$-Arrestin assay (DiscoverX) that measures recruitment of $\beta$-arrestin to the TSHR using enzyme fragment complementation technology. The TSHR and $\beta$-Arr 1 are tagged with two inactive fragments of $\beta$-galactosidase, generating an enzyme donor and enzyme acceptor, and when $\beta$-Arr 1 or $\beta$-Arr 2 translocate to the TSHR, the active $\beta$-galactosidase enzyme is created that can cleave a substrate to generate a chemiluminescent signal (DiscoverX). Two doses of the compounds-11 and $57 \mu \mathrm{M}-$ were used to measure TSHR activation using a fully automated robotic screening system (Kalypsys, San Diego, CA) (Inglese et al., 2006). Briefly, $4.8 \times 10^{3}$ DiscoverX1 cells were seeded with a MultiDrop Combi dispenser (ThermoScientific, Logan, UT) onto white, solid-bottom tissue culturetreated 1536-well plates (Aurora Microplates, Inc., Whitefish, MT) in $3 \mu \mathrm{l}$ of AssayComplete Cell Plating 5 Reagent (DiscoverX) and cultured overnight. Then compounds (as DMSO solutions) were added with a Pintool (PerkinElmer, Waltham, MA) to the assay plates at $23 \mathrm{nl} /$ well. The plates were incubated for 6 hours at ambient temperature followed by addition of $1.5 \mu \mathrm{l}$ PathHunter Detection Reagent per well per the manufacturer's instructions. After 60-minute incubation at ambient temperature, the luminescence signal was measured in a ViewLux uHTS Microplate Imager (PerkinElmer) with 1-minute exposure.

Active compounds (2190) from the primary screen were selected based on their activity $>30 \%$ of basal signal at the lower dose and $>50 \%$ activity of the higher dose. The compounds were retested in the PathHunter $\beta$-Arrestin 1 assay platform described above using a 7-concentration setting in the range of $49 \mathrm{nM}$ to $76 \mu \mathrm{M}$. A parallel counterscreening for cAMP production was performed to select for compounds that activate the $\beta$-arrestin 1-mediated pathway only and did not induce $\mathrm{G}_{\mathrm{s}}$-mediated cAMP production. cAMP production was measured in HEK-TSHR cells in the presence of $50 \mu \mathrm{M}$ Ro-20-1724 (a phosphodiesterase inhibitor) (Sigma-Aldrich). Briefly, $2 \times 10^{3} \mathrm{HEK}-$ TSHR cells/well were seeded onto 1536-well, white, solid-bottom plates in HBSS with $10 \mathrm{mM}$ HEPES and $50 \mu \mathrm{M}$ Ro-20-1724 followed by immediate addition of selected compounds to the cells at $23 \mathrm{nl} /$ well and incubation at $37^{\circ} \mathrm{C}$ for 120 minutes. Subsequently, detection reagents of homogeneous-time-resolved fluorescence cAMP assay (Cisbio, Bedford, MA) were prepared per the manufacturer's instructions, added as a premix in equal volumes of Europium ${ }^{3+}$-cryptatelabeled anti-cAMP antibody and d2 dye-labeled cAMP at $4 \mu \mathrm{l} /$ well, and incubated for 30 minutes at ambient temperature. The signal was measured by EnVision Multilabel Plate Reader (PerkinElmer, Shelton, CT) with excitation at $330 \mathrm{~nm}$ and emission at 620 (donor) and $665 \mathrm{~nm}$ (acceptor). Results were analyzed as the ratio of 665/620 nm multiplied by $10^{4}$.

High-throughput screening data from each assay plate were normalized platewise to corresponding intraplate controls and corrected for systematic deviations in activity values using a combination of control wells within and between the screening plates (Inglese et al., 2006; Southall et al., 2009). Compound concentration-response curves were analyzed, using in-house software, to the standard Hill equation and classified as described previously (Southall et al., 2009). The $\mathrm{EC}_{50}$ 
values of compounds (concentration at $50 \%$ efficacy) were calculated by nonlinear regression analysis using GraphPad Prism software (GraphPad Software, San Diego, CA).

DiscoverX PathHunter $\beta$-Arrestin Assay for Characterization of Hit Compounds. $\beta$-Arr 1 and -2 translocation to the TSHR after receptor activation with the small molecule ligand D3- $\beta$ Arr or bovine TSH (Sigma Aldrich) was measured with the PathHunter $\beta$-Arrestin assay (DiscoverX). Briefly, $1.5 \times 10^{4}$ cells/well were seeded into 96-well plates (Corning Costar \#3610; Sigma Aldrich) in AssayComplete Cell Plating 5 Reagent (DiscoverX) 24 hours before the experiment. DiscoverX1 cells and DiscoverX2 cells were exposed to TSH concentrations between 0 and $10 \mu \mathrm{M}$ and to D3- $\beta$ Arr concentrations between 0 and $100 \mu \mathrm{M}$ for 4 hours at room temperature in AssayComplete Cell Plating 5 Reagent, and afterward the signal ( $\beta$-arrestin 1 or -2 translocation to the TSHR) was detected using the PathHunter Detection Kit per the manufacturer's instructions. The PathHunter CHO-K1 GLP1R $\beta$-Arrestin-1 cells (DiscoverX) were used to test the specificity of the D3- $\beta$ Arr signal at the TSHR.

Measurement of cAMP Production in DiscoverX1 Cells. Cells $\left(5 \times 10^{4}\right.$ per well) were seeded into 48 -well plates. After 24 hours, the cells were assayed for activation (agonist) or inhibition (antagonist) of TSHR signaling in response to added ligands. For measurement of agonistic response, the cells were incubated for 60 minutes in HBSS/10 mM HEPES with $1 \mathrm{mM}$ 3-isobutyl-1-methylxanthine (IBMX; Sigma Aldrich) and 0.18 $\mu \mathrm{M}$ TSH or $10 \mu \mathrm{M}$ D3- $\beta$ Arr in a humidified $5 \% \mathrm{CO}_{2}$ incubator at $37^{\circ} \mathrm{C}$. For measurement of antagonistic response, the cells were incubated with $10 \mu \mathrm{M}$ D3- $\beta$ Arr for 20 minutes in HBSS/10 mM HEPES. After preincubation, the medium was replaced with HBSS/10 mM HEPES containing 1 mM IBMX and $18 \mathrm{nM}$ TSH and $10 \mu \mathrm{M}$ D3- $\beta$ Arr in a humidified $5 \% \mathrm{CO}_{2}$ incubator at $37^{\circ} \mathrm{C}$. After 60 minutes, the incubation was terminated by aspiration of the incubation medium and addition of lysis buffer of the cAMPScreen Direct System (Life Technologies Corporation, Grand Island, $\mathrm{NY}$ ). cAMP content was determined as described by the manufacturer. The chemiluminescence signal was measured in a VICTOR3 V 1420 Multilabel Counter (PerkinElmer).

Measurement of cAMP Production in Primary Cultures of Human Thyrocytes. Thyroid tissue samples were collected from normal thyroid tissue from patients undergoing total thyroidectomy for thyroid cancer at the National Institutes of Health Clinical Center. Patients provided informed consent on an Institutional Review Boardapproved protocol, and materials were received anonymously via approval of research activity through the Office of Human Subjects Research. Primary cultures of human thyrocytes were established from five donors as described previously (Neumann et al., 2011). Thyrocytes $\left(0.8 \times 10^{5}\right.$ cells/well $)$ were seeded into 24 -well plates in Dulbecco's modified Eagle's medium containing 10\% FBS. Twentyfour hours before the experiment, medium was changed to $0.1 \%$ BSA-containing Dulbecco's modified Eagle's medium. Cells were equilibrated for 30 minutes in HBSS/10 mM HEPES, $\mathrm{pH} 7.4$, before stimulation. Cells were pretreated with DMSO (control) or $10 \mu \mathrm{M}$ D3$\beta$ Arr in HBSS/10 mM HEPES for 1 hour. Cells were then stimulated with vehicle or $90 \mathrm{nM}$ TSH ( $\mathrm{EC}_{50}$ for cAMP in human thyrocytes) for 2 hours at $37^{\circ} \mathrm{C}$ in a humidified incubator in the presence of $1 \mathrm{mM}$ IBMX. Incubations were stopped, and the cells were lysed by adding $125 \mu \mathrm{llysis}$ buffer from the cAMP-Screen Direct System. The cAMP content of the cell lysate was determined using the method described in the manufacturer's protocol. The chemiluminescence signal was measured in a VICTOR3 V 1420 Multilabel Counter (PerkinElmer).

Quantitative Real-Time PCR (RT-qPCR). Total RNA was purified using RNeasy Mini Kits (Qiagen Inc., Valencia, CA). First strand cDNA was prepared using a high-capacity cDNA reverse transcription kit (Life Technologies Corporation). RT-PCR was performed in $25 \mu \mathrm{l}$ reactions using cDNA prepared from $100 \mathrm{ng}$ or less of total RNA and TaqMan Universal PCR Master Mix (Life Technologies Corporation). The mRNA expression of osteoblast-specific genes alkaline phosphatase (ALPL) and osteopontin (OPN, gene name SSP1 (secreted phosphoprotein 1)) was measured using primers and probes from Life Technologies Corporation. Quantitative RT-PCR results were normalized to glyceraldehyde 3-phosphate dehydrogenase to correct for differences in RNA input.

Osteopontin Secretion Measurement by ELISA. U2OSTSHR cells were seeded into 24 -well plates at $7 \times 10^{4}$ cells/well. The cells were serum starved in EMEM with $0.1 \%$ BSA 24 hours before addition of TSHR ligands. Thereafter, the cells were incubated with D3- $\beta$ Arr or TSH alone or in combination for 7 days in $0.1 \%$ BSAcontaining EMEM. Cell culture supernatants were used to determine osteopontin (OPN) secretion levels by ELISA (Human OPN Quantikine ELISA; R\&D Systems, Minneapolis, MN) according to the manufacturer's instructions.

Transfection of U2OS-TSHR Cells with siRNA. U2OS-TSHR cells were seeded in EMEM with $10 \%$ FBS into $100 \mathrm{~mm}$ dishes at $1.75 \times 10^{6}$ cells/dish. After 24 hours, the cells were transfected with ON-TARGETplus SMART pool human $\beta$-arrestin 1 or $\beta$-arrestin 2 siRNA or ON-TARGETplus nontargeting pool siRNA using DharmaFECT 1 transfection reagent per the manufacturer's instructions (Thermo Fisher Scientific Inc.). Forty-eight hours after transfection, cells were seeded into 24 -well plates at $7 \times 10^{4}$ cells/ well and incubated overnight. Twenty-four hours later, the cells were treated with TSH with and without D3- $\beta$ Arr in EMEM containing $0.1 \%$ BSA. After 5 days at $37^{\circ} \mathrm{C}$ in a humidified incubator, measurement of OPN secretion was performed as described above.

Data and Statistical Analysis. Data analysis was performed with GraphPad Prism Version 7 for Windows (GraphPad Software). Concentration response data in Figs. 1, 3, and 5 were analyzed with nonlinear regression curve fit, dose-response - stimulation - log (agonist) versus response - variable slope (four parameter) in GraphPad Prism 7. All experimental data are presented as means \pm S.D. The data were analyzed by unpaired two-tailed $t$ test; $* P<0.05$ was considered significant.

\section{Results}

Discovery of a Functionally Selective TSHR Agonist for $\beta$-Arr 1-Mediated Signaling by qHTS. A HTS assay using DiscoverX1 cells allowed us to measure $\beta$-arrestin translocation stimulated by TSHR activation. A total of 368,816 compounds were screened using a fully automated screening system in the qHTS format (Inglese et al., 2006). We identified 975 hit compounds that translocated $\beta$-Arr 1 to the TSHR and that were inactive regarding stimulating TSHRmediated cAMP production. Of these 975 hit compounds, 106 were selected based on potency and efficacy after testing in a nine-point dose response assay in DiscoverX1 cells. We finally selected D3- $\beta$ Arr (Fig. 1A) as the lead compound to study further based on its signaling activity and selectivity for TSHR. The glucagon-like peptide 1 receptor (GLP1R) has been shown to signal through $\beta$-Arr 1 as well (Quoyer et al., 2010). We used an engineered cell line, CHO-K1-GLP1R- $\beta$ Arrestin 1 (DiscoverX), which allows measurement of the translocation of $\beta$-Arr 1 to the GLP1R with the PathHunter $\beta$-Arrestin assay as control to test the specificity of D3- $\beta$ Arr signaling. In contrast to GLP1, D3- $\beta$ Arr did not activate $\beta$-Arr 1 translocation to the GLP1R (Supplemental Fig. 1), a result that is consistent with the idea that D3- $\beta$ Arr initiates translocation of $\beta$-Arr 1 by direct TSHR activation.

The concentration dependence of D3- $\beta$ Arr-stimulated translocation of $\beta$-Arr 1 and -2 compared with their translocation by TSH is illustrated in Fig. 1B. D3- $\beta$ Arr stimulated $\beta$-Arr 1 translocation with an efficacy threefold greater than TSH (Fig. 1B). Although D3- $\beta$ Arr was more effective in translocating $\beta$-Arr 1 than TSH, it was 1.9-fold less effective in 
A

B

DiscoverX1 and DiscoverX2 cells

TSHR- $\beta$-Arrestin-Translocation<smiles>CC(Nc1nc(N2CCNCC2)nc2ccccc12)c1ccccc1</smiles>

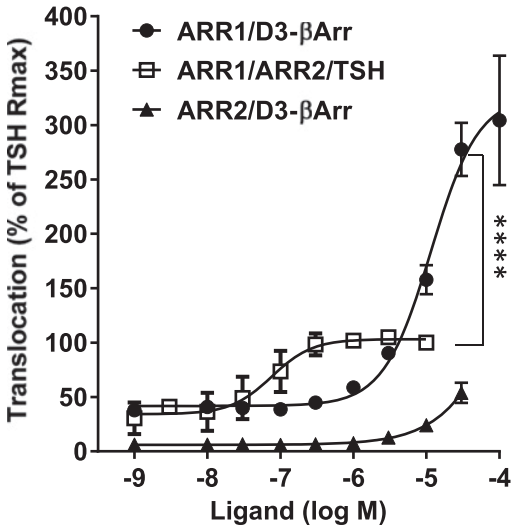

translocating $\beta$-Arr 2 . The $\mathrm{EC}_{50}$ for $\beta$-Arr 1 translocation by TSH was $0.08 \mu \mathrm{M}$ (confidence interval 0.05-0.1 $\mu \mathrm{M}$ ). The $\mathrm{EC}_{50}$ for $\beta$-Arr 1 translocation by D3- $\beta$ Arr was $11.6 \mu \mathrm{M}$ (confidence interval 9.5-14.2 $\mu \mathrm{M}$ ). The translocation assay clearly demonstrated that D3- $\beta$ Arr binds and activates the TSHR. However, D3- $\beta$ Arr had no agonistic effect on cAMP production nor did it inhibit TSH-induced cAMP production in DiscoverX1 cells (Fig. 2, A and B). Noteworthy, D3- $\beta$ Arr did not inhibit TSHinduced cAMP production in primary cultures of human thyrocytes from five donors in which TSHRs are expressed at physiologic levels (Fig. 2C). Therefore, D3- $\beta$ Arr is a functionally selective TSHR agonist toward $\beta$-Arr 1 translocation.

D3- $\beta$ Arr is a Positive Allosteric Modulator for TSH-Induced Translocation of $\boldsymbol{\beta}$-Arr 1 . We found that D3- $\beta$ Arr potentiates the ability of TSH to translocate $\beta$-Arr 1 to the TSHR in DiscoverX1 cells. When we added increasing concentrations of TSH with a constant dose of D3- $\beta$ Arr $(10 \mu \mathrm{M})$, we found that D3- $\beta$ Arr increased the efficacy of TSH in the translocation of $\beta$-Arr 1 at a maximum of $5.1 \pm 0.1$-fold over TSH alone (Fig. 3). Thus, D3- $\beta$ Arr is a positive allosteric modulator (PAM) for TSHR. There was no effect of D3- $\beta$ Arr on $\mathrm{TSH}$ potency. The $\mathrm{EC}_{50}$ s were $64 \mathrm{nM}$ for TSH alone and $62 \mathrm{nM}$ in combination with D3- $\beta$ Arr.

D3- $\beta$ arr is a Positive Allosteric Modulator for TSH-Enhanced Osteoblast Differentiation. We demonstrated previously that $\beta$-Arr 1 plays a role in TSH-mediated upregulation of osteoblast marker genes in vitro (Boutin et al., 2014). Therefore, we next determined if the PAM effect of D3$\beta$ Arr on $\beta$-Arr 1 translocation to the TSHR will translate into potentiation of TSH-induced upregulation of osteoblast-specific genes osteopontin (OPN) and alkaline phosphatase (ALPL). For these experiments, we used U2OS-TSHR cells that are derived from a human osteosarcoma and are a model of osteoblast precursors that we used previously (Boutin et al., 2014, 2016). U2OS cells are frequently used to study the regulation of osteoblastic genes. U2OS-TSHR cells were made to stably express TSHRs as the parental line has only a very low endogenous expression level of TSHR. TSHR mRNAs in parental U2OS cells were $1 / 10,000$ of the level in U2OS-TSHR cells. Treatment of parental U2OS cells with TSH did not result in a physiologic response like cAMP production or increase in osteoblast gene expression. Therefore, the changes in gene expression in U2OS-TSHR cells described below are specific for the TSHR.
Fig. 1. D3- $\beta$ Arr translocates $\beta$-arrestins to the TSHR and is functionally selective toward $\beta$-Arr 1 (A) Two-dimensional structure of D3- $\beta$ Arr [ $N$-(1-phenylethyl)-2-(1-piperazinyl)-4-quinazolinamine; NCGC00379308]. (B) $\beta$-Arrestin translocation to the TSHR was measured with the PathHunter $\beta$-Arrestin assay. DiscoverX1 cells (ARR1) and DiscoverX2 cells (ARR2) were exposed to the indicated concentrations of D3- $\beta$ Arr or TSH for 240 minutes. The dose dependencies of TSH-induced translocation are the same in ARR1 and ARR2 cells, although the absolute levels of fluorescence are different in the two cell lines. We show the levels in the two cell lines as $\%$ of maximum TSH response (TSH Rmax) in each cell line. D3- $\beta$ Arr is more effective in translocating $\beta$-Arr 1 than TSH, but it is less effective in translocating $\beta$-Arr 2 than TSH. The data are from three experiments with duplicate samples and are presented as means \pm S.D. Statistical significance is determined by unpaired two-tailed $t$ test $(* * * * P<0.0001)$.

Figure 4 illustrates the effects of D3- $\beta$ Arr, TSH, or the combination of D3- $\beta$ Arr and TSH on the mRNA levels of these genes in U2OS-TSHR cells. D3- $\beta$ Arr alone did not significantly increase OPN mRNA but induced a $4 \pm 0.6$-fold higher ALPL mRNA level. TSH, as shown previously (Boutin et al., 2014), increased OPN and ALPL mRNA levels in a dosedependent manner. Importantly, D3- $\beta$ Arr potentiated the TSHmediated increase of OPN and ALPL mRNA. At $0.3 \mu \mathrm{M}$ TSH, D3- $\beta$ Arr $(10 \mu \mathrm{M})$ increased OPN mRNA levels from $6 \pm 1$-fold to $325 \pm 37$-fold and ALPL mRNA levels from $21 \pm 1$-fold to
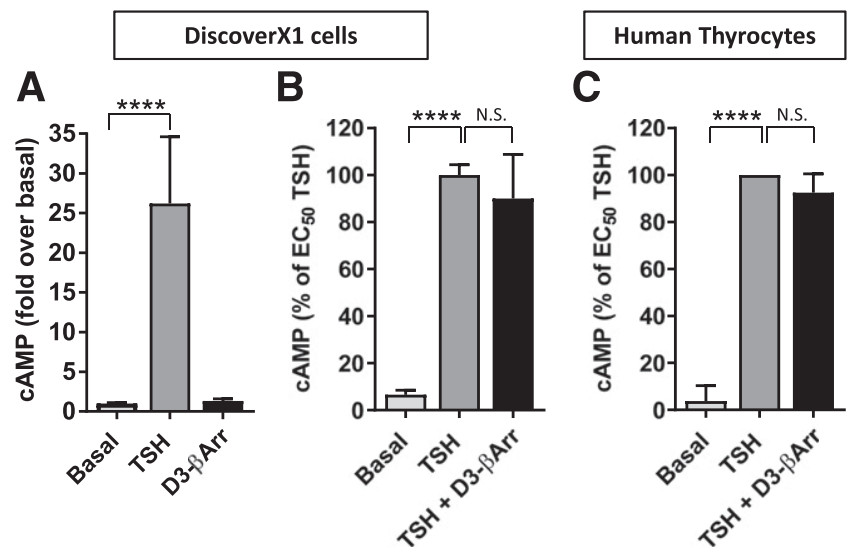

Fig. 2. D3- $\beta$ Arr is a functionally selective TSHR agonist of $\beta$-Arr 1-mediated signaling and does not antagonize TSH-induced cAMP production. (A) DiscoverX1 cells were exposed to $0.18 \mu \mathrm{M}$ TSH or $10 \mu \mathrm{M}$ D3- $\beta$ Arr in HBSS with $1 \mathrm{mM}$ IBMX for 1 hour. cAMP levels were measured by ELISA. In contrast to TSH, D3- $\beta$ Arr-induced activation of the TSHR does not lead to cAMP production. The data are from three experiments with duplicate samples. (B and C) TSH does not antagonize TSHR stimulation of cAMP production. (B) DiscoverX1 cells were pretreated with DMSO (Basal) or $10 \mu \mathrm{M}$ D3- $\beta$ Arr in HBSS for 30 minutes. Subsequently, cells were exposed to $18 \mathrm{nM}$ TSH $\left(\mathrm{EC}_{50}\right)$ with or without $10 \mu \mathrm{M}$ D3- $\beta$ Arr in HBSS with $1 \mathrm{mM}$ IBMX for 1 hour. cAMP levels were measured by ELISA. The data are from three experiments with duplicate samples. (C) Primary cultures of human thyrocytes from five different donors were studied. Cells were pretreated with DMSO (basal) or $10 \mu \mathrm{M}$ D3- $\beta$ Arr in HBSS/HEPES for 1 hour. Subsequently, cells were exposed to $90 \mathrm{nM}$ TSH $\left(\mathrm{EC}_{50}\right.$ for cAMP in human thyrocytes) with or without $10 \mu \mathrm{M}$ D3- $\beta$ Arr in HBSS with $1 \mathrm{mM}$ IBMX for 2 hours at $37^{\circ} \mathrm{C}$. Incubations were stopped, the cells were lysed, and cAMP levels were measured by ELISA. (A-C) The data are presented as means \pm S.D. Statistical significance is determined by unpaired two-tailed $t$ test $(* * * * P<0.0001 ;$ N.S., not significant). 


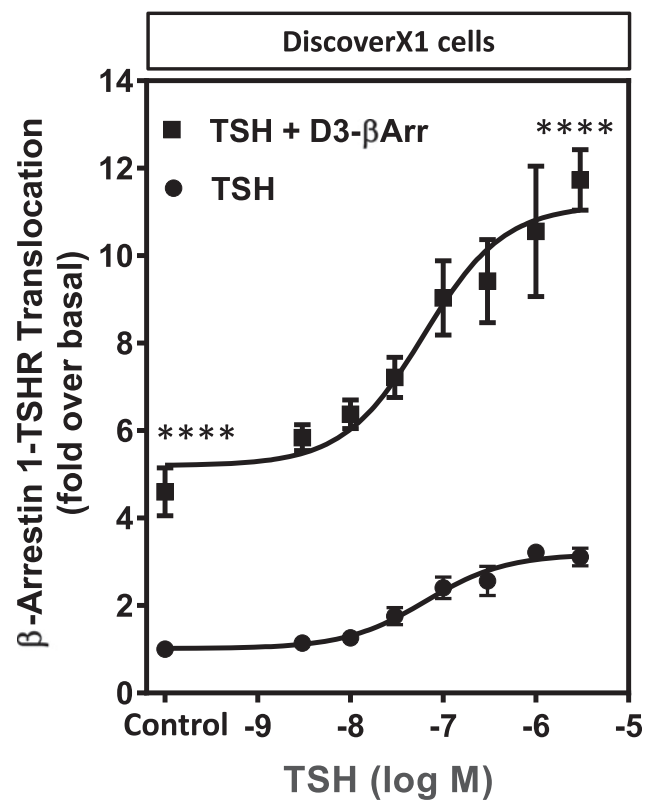

Fig. 3. D3- $\beta$ Arr is a positive allosteric modulator for TSHR- $\beta$-Arr 1 translocation in DiscoverX1 cells. $\beta$-Arr 1 translocation to the TSHR was measured with the PathHunter $\beta$-Arrestin assay. DiscoverX1 cells were exposed to increasing concentrations of TSH with or without $10 \mu \mathrm{M}$ D3- $\beta$ Arr for 240 minutes. D3- $\beta$ Arr potentiates the effect of TSH in translocating $\beta$-Arr 1 to the TSHR. The data are from four experiments with duplicate samples and are presented as means \pm S.D. Statistical significance is determined by unpaired two-tailed $t$ test $(* * * * P<0.0001)$.

$33 \pm 1.4$-fold above control. At $1 \mu \mathrm{M}$ TSH, D3- $\beta$ Arr $(10 \mu \mathrm{M})$ increased OPN mRNA levels from $184 \pm 20$-fold to $1010 \pm$ 111-fold and ALPL mRNA levels from $44 \pm 3.8$-fold to $69 \pm$ 5.4-fold above control.

Furthermore, we confirmed the potentiating effect of D3$\beta$ Arr on the level of OPN protein secretion (Fig. 5). U2OS-TSHR cells were treated with increasing doses of TSH $(0-10 \mu \mathrm{M})$ alone or in combination with 1 or $5 \mu \mathrm{M}$ D3- $\beta$ Arr. Importantly, D3$\beta$ Arr alone had no agonistic effect on OPN secretion. OPN secretion was only increased in the presence of TSH, and D3$\beta$ Arr acted as a potentiator of the TSH-induced effect on OPN secretion in a dose-dependent manner. A low dose of TSH (0.5 $\mu \mathrm{M}$ ) increased OPN secretion $1.7 \pm 0.1$-fold over control, and 1 and $5 \mu \mathrm{M}$ D3- $\beta$ Arr potentiated the TSH effect to increase OPN secretion to $2.5 \pm 0.2$-fold and $3.2 \pm 0.2$-fold above control, respectively. A higher dose of TSH $(5 \mu \mathrm{M})$ increased OPN 21.7 \pm 0.9 -fold, and 1 and $5 \mu \mathrm{M}$ D3- $\beta$ Arr increased OPN secretion further to $31.5 \pm 1.9$-fold and $41.0 \pm 1.7$-fold above control, respectively. Therefore, D3- $\beta$ Arr is a PAM of TSH-enhanced differentiation of osteoblast markers. Noteworthy, D3- $\beta$ Arr does not alter the potency of TSH for OPN secretion. The $\mathrm{EC}_{50}$ s were $1.2 \mu \mathrm{M}$ for TSH alone and 1.2 and $1.1 \mu \mathrm{M}$ for TSH in combination with 1 and $5 \mu \mathrm{M}$ D3- $\beta$ Arr, respectively.

The Potentiating Effect of D3- $\beta$ Arr on TSH-Induced OPN Secretion is Mediated by $\beta$-Arr 1. Since D3- $\beta$ Arr induces translocation of $\beta$-Arr 1 and, to a lesser extent, of $\beta$-Arr 2 to the TSHR, we asked if the effect of this PAM on OPN secretion is mediated by $\beta$-arrestins and if one or both $\beta$-Arr 1 and $\beta$-Arr 2 are involved (Fig. 6). $\beta$-Arr 1 and -2 were knocked down separately in U2OS-TSHR cells using siRNA. RT-qPCR showed a knockdown efficiency of 74.8\% $\pm 5.2 \%$ and $92.4 \% \pm$ $1.2 \%$ for $\beta$-Arr 1 and $\beta$-Arr 2 , respectively (Fig. $6 \mathrm{~A}$ ). In cells treated with scrambled siRNA (control), TSH increased OPN secretion $3.0 \pm 0.2$-fold, and the potentiating effect of D3- $\beta$ Arr on TSH increased the response to $5.3 \pm 0.2$-fold over basal of control. Knockdown of $\beta$-Arr 1 inhibited TSH-induced OPN secretion to $1.9 \pm 0.2$-fold over basal of control $(36 \%$ inhibition), and the synergistic response of TSH and D3- $\beta$ Arr to $2.6 \pm 0.2$-fold over basal of control (51\% inhibition), which is below the level of OPN secretion induced by TSH alone. The silencing of $\beta$-Arr 2 had no effect on OPN secretion (TSH: $2.8 \pm$ 0.3 -fold, TSH and D3- $\beta$ Arr: $5.8 \pm 0.4$-fold over basal of control). The $\beta$-Arr 1 knockdown showed that TSH-induced OPN secretion is also mediated by $\beta$-Arr 1 , and the PAM effect of D3- $\beta$ Arr on TSH-induced OPN secretion appears to be solely mediated by $\beta$-Arr 1 .

\section{Discussion}

In vivo studies in rodents have shown that TSH can prevent bone loss and stimulate bone formation (Abe et al., 2007;

\section{U2OS-TSHR cells}
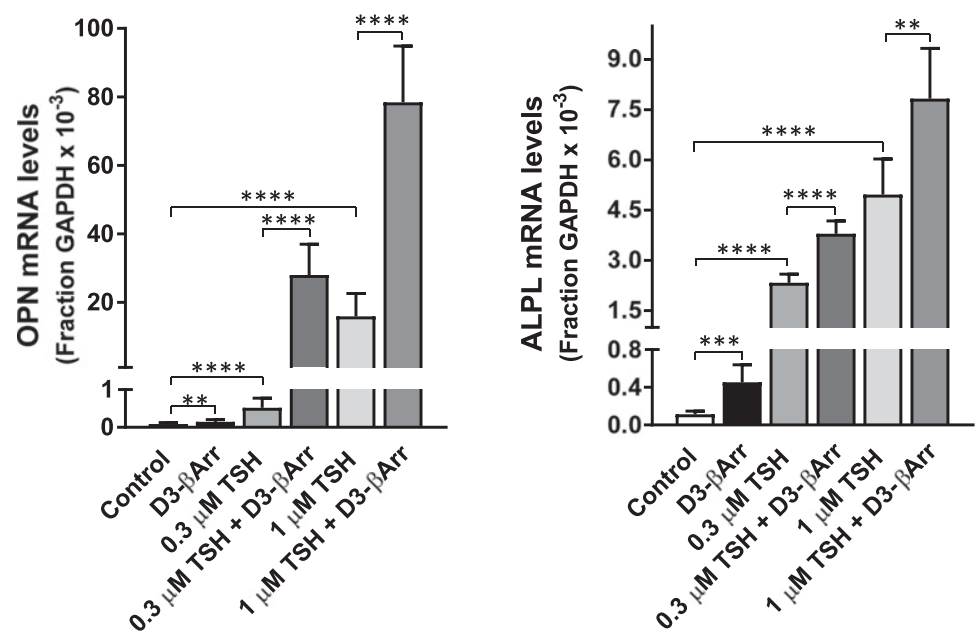

Fig. 4. D3- $\beta$ Arr is a positive allosteric modulator for TSH-induced upregulation of OPN and ALPL mRNAs in U2OS-TSHR cells. Cells were treated with the indicated doses of TSH with and without $10 \mu \mathrm{M}$ D3- $\beta$ Arr in EMEM with $0.1 \%$ BSA. The data represent the gene expression levels of osteoblastic markers OPN and ALPL after 7 days of incubation with ligands. Samples were analyzed by quantitative RT-PCR. The data are from three experiments with duplicate samples and are presented as means \pm S.D. Statistical significance is determined by unpaired twotailed $t$ test $(* * P<0.01$; *** $P<0.001$; **** $P<0.0001)$. 
OPN Secretion in U2OS-TSHR cells

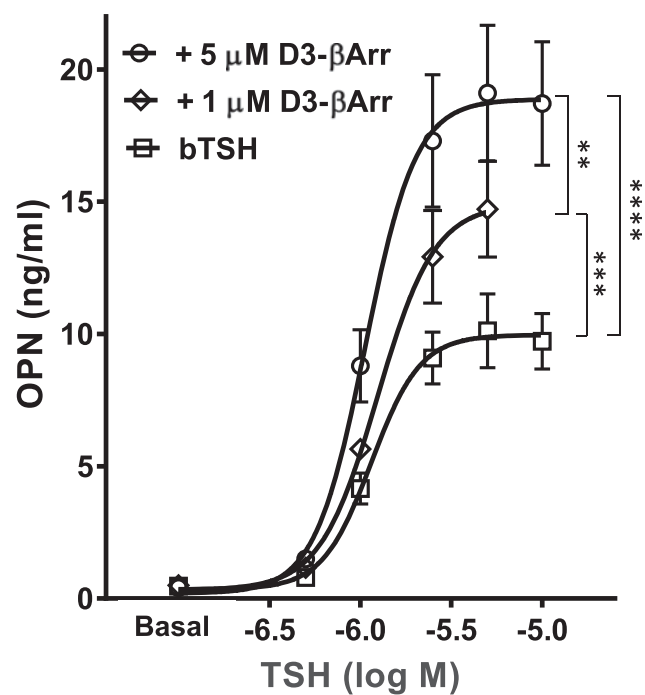

Fig. 5. D3- $\beta$ Arr is a positive allosteric modulator for TSH-enhanced OPN secretion in U2OS-TSHR cells. Cells were treated with increasing doses of TSH with and without 1 or $5 \mu \mathrm{M}$ D3- $\beta$ Arr in EMEM with $0.1 \%$ BSA for 7 days. OPN levels in cell culture supernatants were measured by ELISA The data are from two experiments $(1 \mu \mathrm{M})$ and three experiments $(5 \mu \mathrm{M})$ with duplicate samples and are presented as means \pm S.D. Statistical significance is determined by unpaired two-tailed t test $(* * P<0.01$; $* * * P<0.001 ; * * * * P<0.0001)$.

Sampath et al., 2007; Sun et al., 2008). Based on these studies and others, it has been proposed that these effects may occur by activating TSHRs on preosteoblasts. However, the TSHR-mediated signaling mechanisms in bone are only now being delineated and few studies have used human cells. We, therefore, performed our studies in human-derived preosteoblast-like U2OS-TSHR cells in which wild-type, human TSHRs are stably expressed. It has been known that the TSHR can interact with members of all $G$ protein families (Laugwitz et al., 1996), and we showed previously (Boutin et al., 2014) that TSHR activation by TSH will lead to $\beta$-Arr 1-mediated signaling. Specifically, we showed that TSHR-induced upregulation of osteoblast gene markers in
U2OS-TSHR cells, like PTH effects in bone precursors (GestyPalmer et al., 2009), is mediated by G protein- and $\beta$-arrestinmediated pathways (Boutin et al., 2014, 2016), with TSHR acting via $\beta$-Arr 1 and PTH1R via $\beta$-Arr 2 . We demonstrated that TSH-induced upregulation of interleukin 11 is primarily mediated via $\mathrm{G}_{\mathrm{s}}$-cAMP-protein kinase $\mathrm{A}$, ALPL upregulation is predominantly regulated by activation of $\mathrm{G}_{\mathrm{q} / 11}$-phosphokinase C-ERK1/2 and OPN expression, and secretion is mediated by $\beta$-Arr 1 and $\mathrm{G}_{\mathrm{i}}$-p38 $\alpha$ mitogen-activated protein kinase cascades (Boutin et al., 2016).

Functionally selective GPCR signaling can be caused by ligands that selectively stimulate different signaling pathways leading to distinct physiologic responses. Our finding that TSHR-induced differentiation of U2OS-TSHR cells to osteoblasts is mediated in part by $\beta$-Arr 1 (Boutin et al., 2014) supports the idea that $\beta$-Arr 1 signaling plays a pivotal role in anabolic effects of TSH on bone metabolism. This finding is similar to those induced by the peptidic biased agonist PTH$\beta$ arr, which increases bone formation by activation of PTH1Rmediated $\beta$-Arr 2 signaling (Gesty-Palmer et al., 2009). To study the role of the $\beta$-Arr 1-mediated signaling pathway in TSHR physiology in bone in more detail, we used a HTS to identify a functionally selective agonist of $\beta$-Arr 1 signaling by the human TSHR. We identified the small molecule TSHR agonist D3- $\beta$ Arr (Fig. 1A) and demonstrated that D3- $\beta$ Arr translocates $\beta$-Arr 1 to the TSHR and that it exhibits selective recruitment of $\beta$-Arr 1 over that of $\beta$-Arr 2 (Fig. 1B). D3- $\beta$ Arr did not translocate $\beta$-Arr 1 to the GLP1R in control experiments with CHO-GLP1R- $\beta$ arrestin 1 cells (Supplemental Fig. 1 ), which use the same enzyme fragment complementation technology as DiscoverX1 cells, supporting the idea that D3- $\beta$ Arr directly activates the TSHR. Furthermore, D3- $\beta$ Arr is a functionally selective ligand. It does not stimulate $\mathrm{G}_{\mathrm{s}^{-}}$ mediated signaling (Fig. 2A) nor does it inhibit TSH-induced cAMP production in DiscoverX1 cells overexpressing the TSHR (Fig. 2B) or in primary cultures of human thyrocytes in which the TSHR is expressed at an endogenous level (Fig. 2C). The functional selectivity of D3- $\beta$ Arr allowed us to accentuate the $\beta$-Arr 1-mediated signaling over signaling by $\mathrm{G}$ proteindependent pathways as would have occurred with the native ligand TSH. Indeed, the stronger efficacy for translocation of

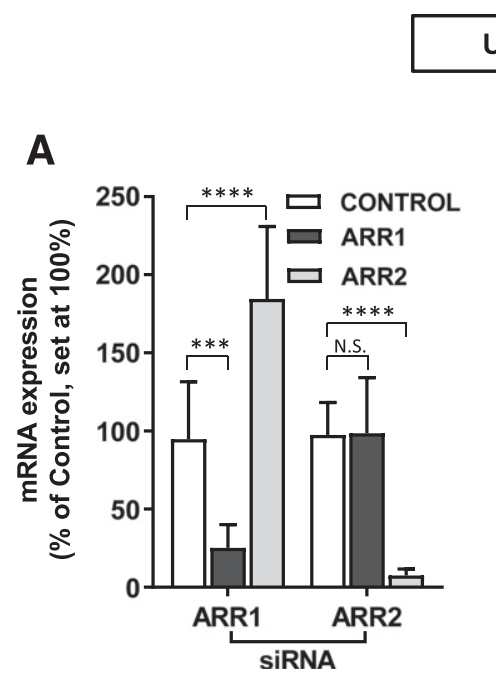

U2OS-TSHR cells

Fig. 6. The potentiating effect of D3- $\beta$ Arr on TSHinduced OPN secretion is mediated by $\beta$-Arr 1 in U2OS-TSHR cells. Cells were transfected with nontargeting (CONTROL), $\beta$-Arr 1 (ARR1), and $\beta$-Arr 2 (ARR2) siRNAs, respectively. (A) Knockdown of ARR1 and ARR2 is demonstrated by RT-PCR. ARR1 and ARR2 mRNAs were measured 72 hours after transfection. The mRNA level in nontreated control cells was set at $100 \%$. The data are from four independent experiments with duplicate samples and are presented as mean \pm S.D. (B) 72 hours after transfection with siRNA, the cells were treated with $1.8 \mu \mathrm{M}$ TSH with and without $10 \mu \mathrm{M}$ D3- $\beta$ Arr in EMEM with $0.1 \%$ BSA for 5 days. OPN secretion was determined by measurement of OPN by ELISA in cell culture medium. The bars represent the means \pm S.D. of four experiments with duplicate samples. Statistical significance is determined by unpaired twotailed $t$ test $\left(* P<0.05 ;{ }^{* *} P<0.01 ; * * * P<0.001\right.$; $* * * * P<0.0001$; N.S., not significant). 
$\beta$-Arr 1 induced by D3- $\beta$ Arr in comparison with TSH (Fig. 1B) might be explained by $\beta$-Arr 1 activation in the absence of potential competition with $\mathrm{G}$ protein(s) for binding to TSHR. The functional selectivity of D3- $\beta$ Arr toward $\beta$-Arr 1 signaling, combined with the fact that D3- $\beta$ Arr does not stimulate nor inhibit TSH-induced cAMP, is promising since $\mathrm{G}_{\mathrm{s}}$-mediated cAMP production is a major player in thyroid hormone synthesis, and therefore, a stimulatory or inhibitory effect of D3- $\beta$ Arr on thyroid metabolism would be unwanted in animals or humans.

As demonstrated in Fig. 1B, D3- $\beta$ Arr alone will induce TSHR-mediated translocation of $\beta$-Arr 1 to the receptor in DiscoverX1 cells. This demonstrates that D3- $\beta$ Arr binds to and activates the TSHR without the presence of TSH. Furthermore, a pivotal finding of this study is that D3$\beta$ Arr potentiated the efficacy of TSH in stimulating $\beta$-Arr 1 translocation to the TSHR (Fig. 3). Because we demonstrated previously the role of $\beta$-Arr 1 -mediated signaling in osteoblast differentiation in vitro, we opted to evaluate the potentiating effect of D3- $\beta$ Arr on bone marker expression. These experiments were carried out in previously established U2OS-TSHR cells that stably express the wildtype, human TSHR and have endogenous wild-type $\beta$-Arr 1 and $\beta$-Arr 2 expression (Boutin et al., 2014). D3- $\beta$ Arr potentiated TSH-mediated upregulation of OPN and ALPL mRNA (Fig. 4) and OPN secretion (Fig. 5). Importantly, D3- $\beta$ Arr alone has no effect on OPN secretion, but it potentiates the efficacy of the orthosteric ligand TSH, thus making the overall response dependent on the presence of the natural ligand and indicating selectivity of D3- $\beta$ Arr toward the TSHR. Therefore, the physiologic rhythms of TSH-TSHR signaling in vivo will be preserved, and adverse effects of the small molecule ligand will be limited. The knockdown of $\beta$-Arr 1 confirmed our previous finding that $\beta$-Arr 1-mediated signaling plays a pivotal role in TSH-induced OPN secretion (Boutin et al., 2014). Recently, we showed that multiple TSHR-activated signaling pathways lead to OPN secretion (Boutin et al., 2016). More importantly, we demonstrated that the potentiating effect of D3- $\beta$ Arr is solely mediated by $\beta$-Arr 1 and not by $\beta$-Arr 2 (Fig. 6B).

In conclusion, we discovered a functionally selective PAM for the TSHR, which, to our knowledge, is the first nonpeptidic PAM to affect TSHR-induced upregulation of osteoblast marker genes in a human in vitro cell model. D3- $\beta$ Arr provides a novel experimental approach to study TSHR-mediated $\beta$-Arr 1 signaling in bone and to understand the functional mechanisms underlying the selective signaling induced by this small molecule TSHR agonist. This PAM or an analog of D3- $\beta$ Arr may be a valuable probe to study TSHR physiology in bone in vivo in future studies.

\section{Authorship Contributions}

Participated in research design: Neumann, Eliseeva, Boutin, Ferrer, Southall, Morgan, Marugan, Gershengorn.

Conducted experiments: Neumann, Eliseeva, Boutin, Barnaeva, Kim, Morgan.

Contributed new reagents or analytic tools: Kim, Marugan.

Performed data analysis: Neumann, Eliseeva, Boutin, Barnaeva,

Ferrer, Southall, Kim, Hu, Morgan, Marugan, Gershengorn.

Wrote or contributed to the writing of the manuscript: Neumann, Gershengorn.

\section{References}

Abe E, Marians RC, Yu W, Wu XB, Ando T, Li Y, Iqbal J, Eldeiry L, Rajendren G, Blair HC, et al. (2003) TSH is a negative regulator of skeletal remodeling. Cell 115: 151-162.

Abe E, Sun L, Mechanick J, Iqbal J, Yamoah K, Baliram R, Arabi A, Moonga BS, Davies TF, and Zaidi M (2007) Bone loss in thyroid disease: role of low TSH and high thyroid hormone. Ann N Y Acad Sci 1116:383-391.

Baliram R, Latif R, Berkowitz J, Frid S, Colaianni G, Sun L, Zaidi M, and Davies TF (2011) Thyroid-stimulating hormone induces a Wnt-dependent, feed-forward loop for osteoblastogenesis in embryonic stem cell cultures. Proc Natl Acad Sci USA 108:16277-16282.

Boutin A, Eliseeva E, Gershengorn MC, and Neumann S (2014) $\beta$-Arrestin-1 mediates thyrotropin-enhanced osteoblast differentiation. FASEB J 28:3446-3455.

Boutin A, Neumann S, and Gershengorn MC (2016) Multiple transduction pathways mediate thyrotropin receptor signaling in preosteoblast-like cells. Endocrinology 157:2173-2181.

Burford NT, Watson J, Bertekap R, and Alt A (2011) Strategies for the identification of allosteric modulators of G-protein-coupled receptors. Biochem Pharmacol 81: 691-702.

Conn PJ, Christopoulos A, and Lindsley CW (2009) Allosteric modulators of GPCRs: a novel approach for the treatment of CNS disorders. Nat Rev Drug Discov 8:41-54.

Coopman K, Huang Y, Johnston N, Bradley SJ, Wilkinson GF, and Willars GB (2010) Comparative effects of the endogenous agonist glucagon-like peptide-1 (GLP-1)(7-36) amide and the small-molecule ago-allosteric agent "compound 2" at the GLP1 receptor. J Pharmacol Exp Ther 334:795-808.

Gentry PR, Sexton PM, and Christopoulos A (2015) Novel allosteric modulators of G protein-coupled receptors. J Biol Chem 290:19478-19488.

Gesty-Palmer D, Flannery P, Yuan L, Corsino L, Spurney R, Lefkowitz RJ, and Luttrell LM (2009) A beta-arrestin-biased agonist of the parathyroid hormone receptor (PTH1R) promotes bone formation independent of G protein activation. Sci Transl Med 1:1ra1.

Gesty-Palmer D and Luttrell LM (2011) 'Biasing' the parathyroid hormone receptor: a novel anabolic approach to increasing bone mass? $B r$ J Pharmacol 164:59-67.

Giusti M, Cecoli F, Ghiara C, Rubinacci A, Villa I, Cavallero D, Mazzuoli L, Mussap M, Lanzi R, and Minuto F (2007) Recombinant human thyroid stimulating hormone does not acutely change serum osteoprotegerin and soluble receptor activator of nuclear factor-kappaBeta ligand in patients under evaluation for differentiated thyroid carcinoma. Hormones (Athens) 6:304-313.

Harrington PE and Fotsch C (2007) Calcium sensing receptor activators: calcimimetics. Curr Med Chem 14:3027-3034.

Huang XP, Karpiak J, Kroeze WK, Zhu H, Chen X, Moy SS, Saddoris KA, Nikolova VD, Farrell MS, Wang S, et al. (2015) Allosteric ligands for the pharmacologically dark receptors GPR68 and GPR65. Nature 527:477-483.

Inglese J, Auld DS, Jadhav A, Johnson RL, Simeonov A, Yasgar A, Zheng W, and Austin CP (2006) Quantitative high-throughput screening: a titration-based approach that efficiently identifies biological activities in large chemical libraries. Proc Natl Acad Sci USA 103:11473-11478.

Karga H, Papaioannou G, Polymeris A, Papamichael K, Karpouza A, Samouilidou E, and Papaioannou P (2010) The effects of recombinant human TSH on bone turnover in patients after thyroidectomy. $J$ Bone Miner Metab 28:35-41.

Kleinau G, Hoyer I, Kreuchwig A, Haas AK, Rutz C, Furkert J, Worth CL, Krause G, and Schülein R (2011) From molecular details of the interplay between transmembrane helices of the thyrotropin receptor to general aspects of signal transduction in family a G-protein-coupled receptors (GPCRs). J Biol Chem 286: $25859-25871$.

Langmead CJ, Austin NE, Branch CL, Brown JT, Buchanan KA, Davies CH, Forbes IT, Fry VA, Hagan JJ, Herdon HJ, et al. (2008) Characterization of a CNS penetrant, selective M1 muscarinic receptor agonist, 77-LH-28-1. Br J Pharmacol 154: 1104-1115.

Latif R, Morshed SA, Zaidi M, and Davies TF (2009) The thyroid-stimulating hormone receptor: impact of thyroid-stimulating hormone and thyroid-stimulating hormone receptor antibodies on multimerization, cleavage, and signaling. Endocrinol Metab Clin North Am 38:319-341, viii.

Laugwitz KL, Allgeier A, Offermanns S, Spicher K, Van Sande J, Dumont JE, and Schultz G (1996) The human thyrotropin receptor: a heptahelical receptor capable of stimulating members of all four G protein families. Proc Natl Acad Sci USA 93:116-120.

Leader A, Ayzenfeld RH, Lishner M, Cohen E, Segev D, and Hermoni D (2014) Thyrotropin levels within the lower normal range are associated with an increased risk of hip fractures in euthyroid women, but not men, over the age of 65 years. $J$ Clin Endocrinol Metab 99:2665-2673.

Martin T, Gooi JH, and Sims NA (2009) Molecular mechanisms in coupling of bone formation to resorption. Crit Rev Eukaryot Gene Expr 19:73-88.

Mazziotti G, Sorvillo F, Piscopo M, Cioffi M, Pilla P, Biondi B, Iorio S, Giustina A, Amato G, and Carella C (2005) Recombinant human TSH modulates in vivo C-telopeptides of type-1 collagen and bone alkaline phosphatase, but not osteoprotegerin production in postmenopausal women monitored for differentiated thyroid carcinoma. J Bone Miner Res 20:480-486.

Mosekilde L, Søgaard CH, Danielsen CC, and Tørring O (1991) The anabolic effects of human parathyroid hormone (hPTH) on rat vertebral body mass are also reflected in the quality of bone, assessed by biomechanical testing: a comparison study between hPTH-(1-34) and hPTH-(1-84). Endocrinology 129:421-428.

Neumann S, Eliseeva E, McCoy JG, Napolitano G, Giuliani C, Monaco F, Huang W, and Gershengorn MC (2011) A new small-molecule antagonist inhibits Graves' disease antibody activation of the TSH receptor. J Clin Endocrinol Metab 96 $548-554$.

O'Brien JA, Lemaire W, Chen TB, Chang RS, Jacobson MA, Ha SN, Lindsley CW, Schaffhauser HJ, Sur C, Pettibone DJ, et al. (2003) A family of highly selective allosteric modulators of the metabotropic glutamate receptor subtype 5. Mol Pharmacol 64:731-740. 
Qin L, Raggatt LJ, and Partridge NC (2004) Parathyroid hormone: a double-edged sword for bone metabolism. Trends Endocrinol Metab 15:60-65.

Quoyer J, Longuet C, Broca C, Linck N, Costes S, Varin E, Bockaert J, Bertrand G, and Dalle S (2010) GLP-1 mediates antiapoptotic effect by phosphorylating Bad through a beta-arrestin 1-mediated ERK1/2 activation in pancreatic beta-cells. $J$ Biol Chem 285:1989-2002.

Rankovic Z, Brust TF, and Bohn LM (2016) Biased agonism: an emerging paradigm in GPCR drug discovery. Bioorg Med Chem Lett 26:241-250.

Rominger DH, Cowan CL, Gowen-MacDonald W, and Violin JD (2014) Biased ligands: pathway validation for novel GPCR therapeutics. Curr Opin Pharmacol 16 108-115.

Salvatori L, Caporuscio F, Coroniti G, Starace G, Frati L, Russo MA, and Petrangeli E (2009) Down-regulation of epidermal growth factor receptor induced by estrogens and phytoestrogens promotes the differentiation of U2OS human osteosarcoma cells. J Cell Physiol 220:35-44.

Sampath TK, Simic P, Sendak R, Draca N, Bowe AE, O’Brien S, Schiavi SC, McPherson JM, and Vukicevic S (2007) Thyroid-stimulating hormone restores bone volume, microarchitecture, and strength in aged ovariectomized rats. J Bone Miner Res 22:849-859.

Siddiqui JA and Partridge NC (2016) Physiological bone remodeling: systemic regulation and growth factor involvement. Physiology (Bethesda) 31:233-245.
Southall NT, Jadhav A, Huang R, Nguyen T, and Wang Y (2009) Enabling the largescale analysis of quantitative high-throughput screening data, in Handbook of Drug Screening (Seethala R and Zhang L eds) pp 442-463, Taylor \& Francis, New York

Sun L, Vukicevic S, Baliram R, Yang G, Sendak R, McPherson J, Zhu LL, Iqbal J, Latif R, Natrajan A, et al. (2008) Intermittent recombinant TSH injections prevent ovariectomy-induced bone loss. Proc Natl Acad Sci USA 105:4289-4294.

Williams GR (2011) Extrathyroidal expression of TSH receptor. Ann Endocrinol (Paris) 72:68-73

Wisler JW, Xiao K, Thomsen AR, and Lefkowitz RJ (2014) Recent developments in biased agonism. Curr Opin Cell Biol 27:18-24.

Wood M, Ates A, Andre VM, Michel A, Barnaby R, and Gillard M (2016) In vitro and in vivo identification of novel positive allosteric modulators of the human dopamine D2 and D3 receptor. Mol Pharmacol 89:303-312.

Wootten D, Christopoulos A, and Sexton PM (2013) Emerging paradigms in GPCR allostery: implications for drug discovery. Nat Rev Drug Discov 12:630-644.

Address correspondence to: Dr. Susanne Neumann, National Institute of Diabetes and Digestive and Kidney Diseases, 50 South Dr., Bldg. 50, Rm. 4130, Bethesda, MD 20892. E-mail: susannen@intra.niddk.nih.gov 